\title{
Drug metabolism and pancreatic cancer
}

\author{
John Paul E. Flores ${ }^{a}$, Robert B. Diasiob, Muhammad Wasif Saifa \\ Tufts Medical Center, Boston, MA; Mayo Clinic, Rochester, MN
}

Abstract

Pancreatic cancer remains a fatal disease in the majority of patients. The era of personalized medicine is upon us: customizing therapy according to each patient's individual cancer. Potentially, therapy can be targeted at individuals who would most likely have a favorable response, making it more efficacious and cost effective. This is particularly relevant for pancreatic cancer, which currently portends a very poor prognosis. However, there is much to be done in this field, and more studies are needed to bring this concept to reality.

Keywords Chemotherapy, genomic profile, genomics, molecular pancreatic pathogenesis, pancreatic cancer pharmacogenomics, pancreatic exocrine cancer, targeted therapy

Ann Gastroenterol 2016; 29 (4): 1-8

\section{Introduction}

Pancreatic cancer, the fourth leading cause of cancerrelated mortality in men and women, continues to carry a poor prognosis, as manifested by a 5 -year survival probability of just $7 \%$ for all comers [1]. In the majority of patients, the presence of extra-pancreatic metastasis at the time of diagnosis precludes surgical resection, and even resected tumors are likely to relapse at either the primary or distant sites.

The long-time mainstay in the treatment of pancreatic cancer has been gemcitabine, shown to be superior to 5-fluorouracil (5-FU) [2]. For several years, this was true for both the adjuvant and the metastatic settings [3]. Several trials attempted to improve upon gemcitabine alone, but they were disappointingly negative [4]. The first improvement was the addition of erlotinib to gemcitabine. This led to only a short, though statistically significant, improvement in overall survival, but it was the first positive trial [5]. Later efforts to ascertain whether K-ras mutations or overexpression of the epidermal growth factor receptor gene were predictive of

${ }^{a}$ Division of Hematology/Oncology and Experimental Therapeutics, Tufts Medical Center, Boston, MA (John Paul E. Flores, Muhammad Wasif Saif); ${ }^{b}$ Department of Molecular Pharmacology and Experimental Therapeutics, Mayo Clinic, Rochester, MN (Robert B. Diasio)

Conflict of Interest: None

Correspondence to: Muhammad Wasif Saif, MD, MBBS, Division of Hematology/Oncology and Experimental Therapeutics, Tufts Medical Center, 800 Washington Street Box: 245 Boston, MA 02111, USA, Tel.: +1 617636 5627, Fax: +1 617636 8538, e-mail: wsaif@tuftsmedicalcenter.org

Received 28 April 2016; accepted 6 July 2016; Published online 21 July 2016

DOI: http://dx.doi.org/10.20524/aog.2016.0074 response (and possibly predictors of a greater clinical benefit) were negative [6]. The next improvements in the metastatic setting were the FOLFIRINOX regimen and gemcitabineabraxane $[7,8]$. Both of these regimens showed a survival benefit when compared to gemcitabine alone, representing the first significant improvements in the field after a series of negative trials. Most recently, the combination of nanoliposomal irinotecan plus 5-FU and folinic acid has shown a survival benefit in the second-line setting after gemcitabine-based therapy [9]. What is not known is how these regimens compare head-to-head, and if certain patients may benefit more from one regimen or another.

A better understanding of the molecular basis of the carcinogenesis of pancreatic cancer, as well as the metabolism of the chemotherapeutic agents, has offered novel therapeutic opportunities. Potentially, new biomarkers may predict the efficacy or toxicity of these agents and aid in the development of novel drugs [10]. Biomarkers are generally considered as predictive or prognostic (Fig. 1). Biomarkers can be measured in tumor tissue or other body fluids, such as plasma.

In this article, the authors review the data on the biomarkers related to the metabolism of these drugs (Table 1) and their and their role in treating patients with pancreatic cancer.

\section{Predictive markers for response to gemcitabine}

\section{Human equilibrative nucleoside transporter 1 (hENT1)}

hENT1 is thought to be the means by which gemcitabine enters tumor cells. Therefore, tumor expression of hENT1 would correlate with the response to gemcitabine, so that low levels of expression would confer relative resistance to gemcitabine. This was first studied clinically in a single-center retrospective study of 21 patients with advanced pancreatic 


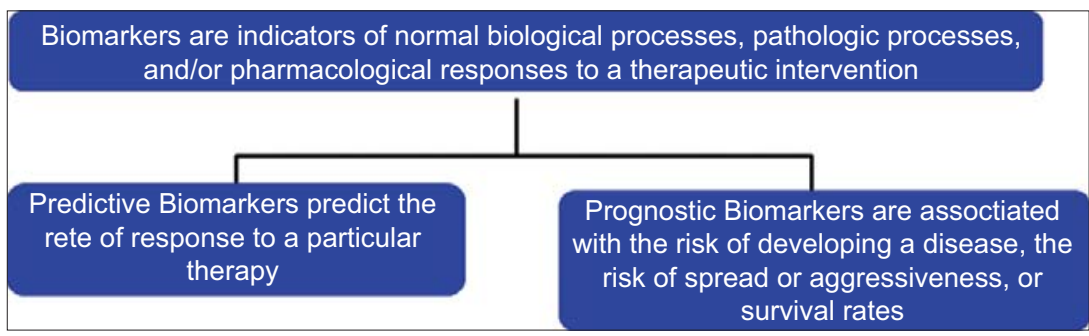

Figure 1 How biomarkers are defined

Table 1 Potential markers and relevant chemotherapeutic agents

\begin{tabular}{ll}
$\begin{array}{l}\text { Chemotherapy } \\
\text { agent }\end{array}$ & Representative candidate gene \\
\hline Gemcitabine & $\begin{array}{l}\text { Cytidine deaminase } \\
\text { Deoxycytidine kinase } \\
\text { Ribonucleotide reductase M1 subunit (RRM1) }\end{array}$ \\
\hline 5-Fluorouracil & $\begin{array}{l}\text { Dihydropyrimidine dehydrogenase (DPD) } \\
\text { Thymidylate synthetase (TS) } \\
5, \text { 10-methylenetetrahydrofolate } \\
\text { reductase (MTHFR) }\end{array}$ \\
Irinotecan & $\begin{array}{l}\text { UDP glucuronosyltransferase 1 family, } \\
\text { polypeptide A1 (UGT1A1) }\end{array}$ \\
Cisplatin & $\begin{array}{l}\text { Excision repair cross complementation } \\
\text { group 1 and 2 (ERCC1 and ERCC2) }\end{array}$ \\
Oxaliplatin & $\begin{array}{l}\text { Excision repair cross complementation } \\
\text { group 1 (ERCC1) }\end{array}$ \\
Erlotinib & $\begin{array}{l}\text { Epidermal growth factor receptor (EGFR) } \\
\text { K-ras }\end{array}$ \\
\hline Nab-paclitaxel & $\begin{array}{l}\text { Secreted protein acidic and rich in } \\
\text { cysteine (SPARC) }\end{array}$ \\
\hline
\end{tabular}

adenocarcinoma treated with gemcitabine alone [11]. hENT1 was measured by immunohistochemistry (IHC) and scored using relative staining intensity in tumor cells compared to islets of Langerhans cells and lymphocytes. Nine of 21 patients with uniform staining had a median overall survival of 13 months, compared to 4 months in the 12/21 who had areas of absent staining. This study was followed by several other retrospective studies evaluating hENT1 expression in patients treated with gemcitabine [12]. The methods of hENT detection varied, some using polymerase chain reaction (PCR), and IHC being scored differently, but these studies all found that higher hENT expression corresponds to improved survival under treatment with gemcitabine.

There have also been several post-hoc analyses of prospective cohorts using hENT1. Ferrell et al performed a post-hoc analysis of the RTOG 9407 trial [13]. In this trial, all patients had resected pancreatic adenocarcinoma treated with adjuvant chemoradiation and 5-FU. They were randomized to receive gemcitabine or 5-FU both before and after chemoradiation. Tissue from 198 of the trial's 538 patients was assayed for hENT1 by IHC. hENT1 was scored as no staining, low or high, seen in $22 \%, 40 \%$ and $37 \%$ of patients respectively. Amongst the patients in the gemcitabine arm, those with high or low
hENT1 staining showed a survival benefit compared to no staining. There were no statistically significant differences in survival based on hENT1 staining in the 5-FU arm.

Greenhalf et al performed a post-hoc analysis of the ESPAC-3 trial using hENT1 [14]. ESPAC-3 was an adjuvant trial of 5-FU vs. gemcitabine vs. observation, though the observation arm was later removed. In the trial, gemcitabine was found to be non-inferior to 5-FU in the adjuvant setting [15]. hENT1 was scored as either high or low, defined as above or below median. Patients with high hENT1 expression who received gemcitabine had better survival compared to those with low hENT1 expression (median overall survival 26.2 vs. 17.1 months), while hENT1 expression had no effect on survival among those who received 5-FU (median overall survival 21.9 vs. 25.6 months in the high and low groups, respectively). The authors concluded that, in the adjuvant setting, patients with low expression of hENT1 should not receive gemcitabine and that $5-\mathrm{FU}$ is a more effective alternative.

There has been one prospective trial using hENT1. The LEAP trial was a prospective Phase II trial in patients with metastatic pancreatic adenocarcinoma. It compared gemcitabine to CO101 , a gemcitabine variant in which gemcitabine is covalently bonded to elaidic acid, designed to enter tumor cells despite low hENT1 [16]. A total of 367 patients were enrolled and hENT1 expression was determined in $97.5 \%$ of them using IHC. Although the investigators used a novel hENT1 antibody and scoring methodology, they validated their methods in a separate post-hoc analysis of tissue from 225 patients in the RTOG 9407 study, as well as a separate retrospective analysis of gemcitabine-treated patients, which yielded results that were concordant with those of prior studies. Surprisingly, there was no difference in overall survival associated with treatment or hENT1 expression level, even amongst those treated with gemcitabine. A further study that compared only those with very high hENT1 staining versus no staining also failed to show a difference. The reasons for these negative outcomes are unclear, especially in the context of prior studies. The major differences in the trial were the different antibody and scoring system, as mentioned above, and the fact that it was focused on metastatic disease, whereas most previous studies involved an adjuvant population.

An additional post-hoc study was carried out in the metastatic setting, using data and tissue from the AIO-PK0104 study [17]. This was a Phase III study of advanced pancreatic adenocarcinoma, in which patients were treated with either gemcitabine and erlotinib followed by capecitabine, or capecitabine and erlotinib followed by gemcitabine [18]. In 
the post-hoc analysis, hENT1 expression was determined and quantified by the same method used in the LEAP trial. Similarly, the investigators did not find a correlation between survival and hENT1 expression levels amongst the study groups. There were in fact some conflicting data, as high hENT1 expression was found to correlate with a shorter time to first treatment failure in patients randomized to gemcitabine and erlotinib as first-line treatment.

Taken together, retrospective studies indicate that hENT1 can be used as a predictive biomarker of gemcitabine response, but prospective studies have not confirmed this. Additionally, it appears to be a better marker in the adjuvant setting, perhaps indicating that the marker is less useful in the setting of increased heterogeneity of metastatic disease.

\section{Deoxycytidine kinase (dCK)}

$\mathrm{dCK}$ is an enzyme that catalyzes the rate-limiting step in the metabolism of gemcitabine to its active form. Thus, intratumoral levels of expression may be a prognostic factor in patients treated with gemcitabine. The first analysis of this was by Sebastiani et al, who analyzed 44 patients with metastatic pancreatic cancer retrospectively [19]. They found that most cancers had some staining, with absence in only 4 samples. In 32 patients in whom complete treatment and follow up were known, they found that high dCK immunostaining correlated with longer overall survival in those patients treated with gemcitabine.

Maréchal et al did a similar analysis of dCK in an adjuvant cohort of 45 patients enrolled in various trials at their institution, who were treated with gemcitabine and gemcitabine-based chemoradiation [20]. They split their cohort into halves of low and high staining; the latter group had longer overall survival. In an attempt to assess the value of dCK and hENT1 in a larger cohort, a study of 572 consecutive patients with resectable pancreatic cancer treated with resection was conducted at 5 centers [21]. Of these, 434 were evaluated, while some were removed because of inclusion in prior studies or incomplete follow-up data; 243 of these patients received adjuvant gemcitabine. In concordance with prior studies, high levels of hENT1 and dCK were associated with longer overall survival in those treated with gemcitabine. There were no correlations seen in the groups of patients who were treated with surgery alone or who did not receive gemcitabine. Though these studies of dCK have shown consistency in both the adjuvant and metastatic settings, prospective studies are needed to confirm its value as a prognostic or predictive marker.

\section{Predictive markers for response to platinum agents}

\section{Excision repair cross-complementation group 1 (ERCC1)}

ERCC1 gene encodes the DNA excision repair protein ERCC1, whose crucial role in nucleotide excision repair is thought to promote relative resistance to platinum agents. In pancreatic adenocarcinoma, it was first studied by Akita et al in a retrospective cohort of 68 patients who underwent resection [22]. They were divided into high and low groups by IHC, based on median expression. Those with high ERCC1 expression were found to have a survival benefit compared to those with low expression. Similarly, high expression of ribonucleotide-reductase subunit-1 (RRM1) was also found to be associated with longer survival. In this study, among 28 patients treated with gemcitabine, those with low RRM1 expression showed a survival benefit.

Valsecchi et al performed a similar study of ERCC1 and RRM1 [23] in a retrospective cohort of 94 patients with resected pancreatic adenocarcinoma. Their population differed in that $87 \%$ received adjuvant gemcitabine. In contrast to the prior study, they found no associations between ERCC1 or $R R M 1$ expression and overall survival.

Maithel et al studied ERCC1 expression in the first-line adjuvant setting [24]. They carried out a retrospective study of 95 patients and measured ERCC1 by IHC. High expression was found in $16 \%$ of cases and was found to be associated with shortened median overall and recurrence-free survival. The same applied to the proportion of patients who underwent adjuvant treatment $(n=73)$. Most patients had received 5-FU or gemcitabine, but details of chemotherapy were not available.

Mancuso et al studied a retrospective cohort of patients receiving second-line treatment with 5-FU, either alone $(n=50)$ or in combination with a platinum agent $(n=58)$ [25]. All patients had previously failed a gemcitabine-containing regimen. ERCC1 expression was measured by IHC and reverse transcription (RT)-PCR from archived tumor samples, and the authors found both methods to be highly concordant. In patients treated with platinum, those with low ERCC1 expression were found to have longer overall survival than those with high ERCC1 expression. No difference in overall survival was seen in the patients treated with 5-FU alone.

Fuereder et al evaluated 26 patients for ERCC1 expression in a retrospective cohort of metastatic pancreatic cancer patients who received first line gemcitabine, oxaliplatin, and erlotinib [26]. Their study was not powered to detect survival differences, but they did find that those who responded to the regimen were more likely to have high ERCC1 expression. The reason for this unexpected result is not clear. From these studies, the data on ERCC1 are mixed. This perhaps reflects the differences in the study populations and treatments, though a consistent effect is difficult to parse from these inconsistent data.

\section{Predictive markers for response to 5-FU}

\section{Thymidylate synthase (TS)}

One of the targets of 5-FU is TS, the inhibition of which would therefore inhibit DNA synthesis. It is postulated that the level of TS present would correlate with sensitivity to 5-FU or derivatives thereof, and tumors with relatively high levels 
present would be more sensitive to 5-FU. The typical assay is an IHC test that can be performed on standard paraffin blocks. However, it is not standardized, and different investigators have used varying thresholds.

The first study of TS in pancreatic adenocarcinoma was by Takamura et al [27]. They performed a retrospective singlecenter study of 102 patients who underwent surgery for pancreatic cancer from 1980-2000. They found TS expression was "high" (over $25 \%$ staining by IHC) in $44 \%$ of their samples taken from both primary and metastatic sites. Resectable patients were found to do better with high levels of TS, with or without 5-FU-based chemotherapy. Among unresectable patients, those who had high TS expression were found to have a greater benefit from 5-FU-based chemotherapy.

Another study by $\mathrm{Hu}$ et al evaluated TS expression retrospectively in 138 patients who underwent resection at two centers [28]. TS was evaluated by IHC and classified as low or high; it was found to be high in $63 \%$. In this study, high TS was a poor prognostic marker. Among the 73 patients who received 5-FU adjuvant therapy, those with high TS expression had a reduced risk of death, whereas 5-FU did not influence the risk of death amongst those with low TS expression.

A study by Formentini et al also evaluated TS [29]. This study was also a single-institution retrospective study of 138 patients. High TS expression was defined as $2+$ or higher on a scale of $0-3$ and was found in $43 \%$ of this population. Among the entire cohort, TS expression was not a prognostic factor. In those undergoing resection $(\mathrm{n}=98)$, high TS expression was associated with lower survival. TS expression did not have a statistically significant effect on survival in treatment subgroups, but a trend toward better survival in 23 patients treated with intra-arterial 5-FU based chemotherapy was seen for those with low levels of TS. The authors postulate that those with high TS levels are relatively resistant because of insufficient inhibition by 5 -FU.

Van der Zee et al also studied TS expression in pancreatic cancer [30]. This was a retrospective single-center study of 212 patients who underwent R0 resections of a pancreatic adenocarcinoma originating in either the head of the pancreas or the ampulla of Vater. TS levels were determined from resection specimens by IHC and considered "high" if cytosolic expression was seen in at least $50 \%$ of tumor cells. High TS expression was detected in $26 \%$ of pancreatic head tumors and $43 \%$ of periampullary tumors; it was found to correlate with improved survival in the pancreatic head group, but had no significant effect in the periampullary group. The authors did not report any correlation with 5-FU-based treatment, as the number of patients was insufficient.

All four studies were performed retrospectively in one or two centers, with a limited patient population and over several years, in heterogeneously treated patients with variations in TS evaluation. Despite those limitations, the results suggest that TS could be used as a prognostic marker and has the potential to be a predictive marker as regards the response to 5-FUbased therapy. Data from a more homogeneous prospective dataset are needed, while prospective data will eventually be needed as validation.

\section{Dihydropyrimidine dehydrogenase (DPD)}

DPD is a key enzyme in the degradation of 5-FU. It is thought that intratumor expression of DPD may correlate with possible 5-FU resistance. The first study of DPD in pancreatic cancer was a retrospective analysis by by Nakayama et al that included 68 patients that included 68 patients with resectable pancreatic adenocarcinoma, 30 of whom received $5-\mathrm{FU}$ via a liver perfusion technique [31]. They measured DPD by IHC and found that those with negative staining had longer survival if treated with intrahepatic 5-FU, compared to those with positive staining. Intrahepatic 5-FU had no effect on survival in the DPD positive group.

DPD was also studied by Kuramochi et al in patients receiving S-1 [32]. The investigators evaluated DPD expression measured by RT-PCR in patients with recurrent pancreatic cancer. In 33 patients treated with S-1 and cisplatin after recurrence, they found that patients who responded to therapy (as measured by CA 19-9) had lower mean intratumoral DPD expression.

In a larger study of DPD in the adjuvant setting, Kondo et al [33] retrospectively evaluated 106 patients treated with adjuvant therapy. They observed high DPD expression in $37 \%$ of patients, as measured by IHC. Those with low DPD expression were found to have better median overall survival if they received S-1 (all these patients also received gemcitabine). No differences in survival were seen based on treatment in the high DPD group, or in the group not treated with S-1 when analyzed according to DPD expression. Interestingly, the investigators also evaluated TS expression and did not find any correlation with overall survival by treatment.

A similar study by Kondo et al evaluated both DPD and hENT1 by IHC in 86 patients treated with adjuvant S- 1 and gemcitabine [34]. Low DPD and high hENT1 expression were observed as favorable factors in this study. Those with one or two favorable factors had an overall survival benefit when compared to those patients with no favorable factors. Like TS, DPD may be a useful marker for guiding therapy with 5-FU based treatments, but prospective data are lacking for validation.

\section{Microsatellite instability (MSI)}

Studies have also suggested that 5-FU may be less effective in patients with microsatellite instability (MSI) pancreatic cancer; these findings resemble data seen in the treatment of colon cancer, especially in the adjuvant setting. In a study by Nakata et al, 46 patients with pancreatic cancer who had undergone resection were evaluated for microsatellite variations [35]. Univariate analysis showed that patients with MSI-positive tumors had significantly longer survival than those with MSInegative tumors, although there were no significant differences in clinicopathological factors between the two groups (median survival 62 vs. 10 months, respectively; $\mathrm{P}=0.011$ ). Multivariate survival analysis indicated that MSI status had an independent 
predictive value (hazard ratio 5.577; $\mathrm{P}=0.007$ ). The tumorinfiltrating leukocyte intensity was stronger in MSI-positive tumors than in MSI-negative tumors, suggesting that MSIpositive tumors may induce stronger antitumor immunity. However, more data will be required to confirm the role of MSI as a marker in these patients.

\section{Other biomarkers}

\section{Histone modifications}

Histone modifications were identified as a potentially useful biomarker in pancreatic cancer by Wei et al, who found that low levels of trimethylation at lysine 27 of histone H3 (H3K27me3), measured by IHC, corresponded to a poor prognosis in a retrospective cohort with pancreatic cancer [36]. This was followed by a single-center retrospective study of 140 Stage I-II patients and a post-hoc analysis of 195 patients enrolled in the RTOG 9407 trial by Manuyakorn et al, assessing for histone modifications [37]. The investigators chose to assess histone $\mathrm{H} 3$ lysine 4 dimethylation (H3K4me2), histone $\mathrm{H} 3$ lysine 9 dimethylation (H3K9me2), and histone $\mathrm{H} 3$ lysine 18 acetylation (H3K18ac). Both populations studied were treated with resection followed by adjuvant treatment. In RTOG 9407, patients were randomized to adjuvant 5-FU or gemcitabine before and after chemoradiation with 5-FU. The authors found that, in general, low levels of any of the histone modifications correlated with a worse prognosis. In RTOG 9407, low $\mathrm{H} 3 \mathrm{~K} 4 \mathrm{me} 2$ or low $\mathrm{H} 3 \mathrm{~K} 9 \mathrm{me} 2$ were independent predictors of worse overall survival. In the retrospective cohort of 140 Stage I-II patients, all three of the modifications were associated with worse overall survival. Additionally, these associations were stronger if the cohorts studied were limited to node-negative patients. Finally, in the RTOG9407 cohort, it was found that low $\mathrm{H} 3 \mathrm{~K} 4 \mathrm{me} 2$ or low $\mathrm{H} 3 \mathrm{~K} 9 \mathrm{me} 2$ was associated with worse overall survival in the subgroup that received adjuvant 5-FU, but had no effect in those that received gemcitabine. This suggests that histone modifications could potentially be used as a predictive marker.

Another retrospective study of several histone modifications was performed in a Japanese population who were treated with resection and who were all offered adjuvant gemcitabine [38]. The researchers measured the expression of dimethyl histone H3 lysine 9 (H3K9me2), acetyl histone H3 lysine 9 (H3K9ac), dimethyl histone $\mathrm{H} 3$ lysine 4 (H3K4me2), trimethyl histone H3 lysine 4 (H3K4me3), and acetyl histone H3 lysine 18 (H3K18ac) by IHC, and classified them as high- or low-score based on expression strength and percentage of cells stained. Though various histological features were correlated with different histone modification expression levels, there were no correlations with disease-free survival or overall survival. However, low H3K4me2 expression was associated with decreased disease-free survival in patients who completed adjuvant gemcitabine. No patients were treated with 5-FU in this cohort. Histone modifications may be a prognostic marker, but it is still unclear whether they could be used to guide treatment as a predictive marker. The ideal set of modifications to follow remains unclear.

\section{Secreted protein acidic and rich in cysteine (SPARC)}

SPARC is a matricellular protein that modulates cell adhesion and proliferation. Barriers to pancreatic tumor drug delivery, including the desmoplastic reaction, have gained a great deal of attention over the last decade. It is believed that SPARC is among the molecules responsible for this dense stroma around the cancer cells in the pancreas. In addition, SPARC has other duties, including wound healing, cell adhesion, signaling, angiogenetic inhibition, epithelial-to-mesenchymal transition, and acting as a tumor suppressor gene[39].

Studies have found that high SPARC mRNA expression was a significant independent prognostic marker for pancreatic cancer, with the five-year survival rate of patients with a low SPARC mRNA level at $20 \%$ compared with $0 \%$ for patients with a high SPARC mRNA level [40]. A previous study explored the role of SPARC in drug delivery and compared nab-paclitaxel combined with gemcitabine to gemcitabine alone in mice. The results showed that the nab-paclitaxel mouse group had increased intratumoral concentrations of gemcitabine and decreased peritumoral desmoplastic stroma. This suggests that nab-paclitaxel may target stromal SPARC and allow the delivery of chemotherapy to the targeted tumor [41]. As previously mentioned, in the phase III MPACT trial the combination of nab-paclitaxel plus gemcitabine was superior to gemcitabine alone for patients with metastatic pancreatic cancer [8]. In addition to efficacy parameters, this study also assessed the association of SPARC levels with efficacy as an exploratory endpoint. Recently Hidalgo et al published the final analysis, in which they found no association between stromal SPARC level and overall survival in either treatment arm. Neither tumor epithelial SPARC nor plasma SPARC was associated with overall survival; hence, the study did not support any treatment decisions based on SPARC expression regarding nab-paclitaxel plus gemcitabine or gemcitabine alone [42].

\section{BRCA2 mutations}

BRCA2 mutations are found in up to $17 \%$ of patients with familial pancreatic cancer. The protein product of the BRCA2 gene plays an important role in the repair of DNA cross-linking damage. It is located on chromosome $13 \mathrm{q}$ and is inactivated in fewer than 10 percent of pancreatic cancers. The gene is almost always inactivated by a germline (inherited) mutation coupled with somatic loss of the second allele [43]. It has been suggested that this function of BRCA2 can be exploited therapeutically. In vitro studies suggest that pancreatic cancers with genetically inactivated $B R C A 2$ are significantly more susceptible to DNA cross-linking agents than are pancreatic cancers with a genetically intact BRCA2. Indeed, several reports have documented remarkable therapeutic responses 
to DNA cross-linking agents, such as mitomycin or cisplatin, or to poly ADP-ribose polymerase inhibitors in patients whose cancers have inactivated BRCA2 [44,45].

\section{Uridine diphosphate-glucuronosyltransferase (UGT1A1)}

Irinotecan, as part of the FOLFIRINOX regimen, or as a single agent, or with 5-FU (FOLFIRI), is used by oncologists to treat patients with pancreatic cancer. A novel form of irinotecan, nanoliposomal irinotecan, combined with 5-FU and folinic acid, has recently been approved by FDA for the second-line treatment of patients who have failed gemcitabinebased chemotherapy [46]. After intravenous administration, irinotecan is converted by a carboxylesterase to its active metabolite, 7-ethyl-1 o-hydroxycamptothecin (SN-38). SN-38 is then detoxified by UGT1A1 enzyme to its inactive form SN38 glucuronide (SN-38G), excreted into the bile and urine. UGT1A1 has been found to be polymorphic, and results in a wide inter-individual variation in patient responses, as well as toxic side-effects. Based on this information, FDA first approved the irinotecan label with pharmacogenetic information in 2005. In 2010, new pharmacogenetic information was added to the label regarding the risk of neutropenia in patients who had a genetic defect of UGT1A1.

Over 30 genetic variants in the promoter region and exon 1 of UGT1A1 that can decrease enzyme activities have been identified. These genetic variants have been linked to a few syndromes, such as Gilbert's and Crigler-Najjar syndromes. UGT1A $1{ }^{\star} 28$ has been reported to cause an approximately $70 \%$ reduction in UGT1A1 enzyme activity. Underexpression of UGT1A1 enzyme impairs the metabolism of SN-38 to its inactive form (SN-38G) and causes an excessive accumulation of toxic SN-38.

The French Joint Workgroup developed a review of theimpact of the deficient UGT1A1 ${ }^{\star} 28$ variant on irinotecan efficacy and toxicity [47]. In addition, there are inter-ethnicity variations. Therefore, physicians caring for these patients should be aware of these genetic abnormalities. The practical point is that we normally administer irinotecan doses at least equal to $180 \mathrm{mg} /$ $\mathrm{m}^{2}$, but this dose should be reduced in patients who are known to be homozygous for the UGT1A1*28 allele.

\section{Potential future biomarkers}

While none of the markers mentioned above has sufficient data to support incorporation into practice, each has shown the potential to be useful. It may be that any one marker in isolation is not sufficiently predictive, whereas several markers could be used in combination. For example, a panel of several markers for gemcitabine and 5-FU could be used to determine whether gemcitabine/nab-paclitaxel or FOLFIRINOX should be used as first-line treatment. The ability to run these tests using standard IHC techniques makes this concept feasible.
Pancreatic cancer may benefit from advances made in other tumor types, as predictive markers are being sought across the board. The prospect of immunotherapy is exciting in pancreatic cancer [48]. Though studies showing efficacy have yet to be completed, prospective markers such as programmed death-ligand 1 (PD-L1) expression, mutational status, or tumor-infiltrating $\mathrm{T}$ cells in other cancers seem promising, and progress found in other tumor types could be easily translated. Additionally, improvements in the cost and efficiency of next-generation sequencing techniques allow for broad-based screens of a tumor genome or determination of a tumor's transcriptome. Though this technology is available, we do not yet know how best to use it and how to incorporate the information into regular practice. Furthermore, predictive markers may be assayed from circulating tumor cells or circulating free tumor DNA [49]. One advantage of this is, of course, ease, as tissue can often be limited. More importantly, such "liquid biopsies" may better account for tumor heterogeneity, as a tissue sample will not be limited to one site.

\section{Concluding remarks}

Pancreatic cancer remains a difficult disease with a poor prognosis. The rarity of the disease makes it difficult to screen for effectively, and the lack of clear standards of care internationally compounds efforts toward improvement. The general lack of effective treatment options made the concept of predictive biomarkers moot, but with recent improvements in therapy there is now a need. There are a host of potential biomarkers that may be useful in predicting the response to 5-FU and gemcitabine-based treatments. There are also unique targets such as stromal-targeted therapy. Biomarkers will also need to be validated across disease stage, as the findings in the adjuvant and metastatic settings appear to differ. Improvements will potentially come from efficiently designed Phase III trials, which inherit a strong basis from randomized Phase II studies and, ideally, will address at least two clinical questions effectively in one trial, such as simultaneous prospective validation of a biomarker coupled with a treatment decision.

\section{References}

1. Siegel RL, Miller KD, Jemal A. Cancer Statistics, 2016. CA Cancer J Clin 2016;66:7-30.

2. Burris HA $3^{\text {rd }}$, Moore MJ, Andersen J, et al. Improvements in survival and clinical benefit with gemcitabine as first-line therapy for patients with advanced pancreas cancer: a randomized trial. J Clin Oncol 1997;15:2403-2413.

3. Saif MW. Controversies in the adjuvant treatment of pancreatic adenocarcinoma. JOP 2007;8:545-552.

4. Saif MW. Advanced stage pancreatic cancer: novel therapeutic options. Expert Rev Clin Pharmacol 2014;7:487-498.

5. Moore MJ, Goldstein D, Hamm J, et al. Erlotinib plus gemcitabine compared with gemcitabine alone in patients with advanced pancreatic cancer: a phase III trial of the National Cancer Institute 
of Canada Clinical Trials Group. J Clin Oncol 2007;25:1960-1966.

6. Boeck S, Jung A, Laubender RP, et al. EGFR pathway biomarkers in erlotinib-treated patients with advanced pancreatic cancer: translational results from the randomised, crossover phase 3 trial AIO-PK0104. Br J Cancer 2013;108:469-476.

7. Conroy T, Desseigne F, Ychou M, et al. FOLFIRINOX versus gemcitabine for metastatic pancreatic cancer. $N$ Engl $J$ Med 2011;364:1817-1825.

8. Von Hoff DD, Ervin T, Arena FP, et al. Increased survival in pancreatic cancer with nab-paclitaxel plus gemcitabine. $N$ Engl $J$ Med 2013;369:1691-1703.

9. Wang-Gillam A, Li CP, Bodoky G, et al. Nanoliposomal irinotecan with fluorouracil and folinic acid in metastatic pancreatic cancer after previous gemcitabine-based therapy (NAPOLI-1): a global, randomised, open-label, phase 3 trial. Lancet 2016;387:545-557.

10. Kim R, Mahipal A, Choi M, Saif MW. Biomarkers for pancreatic cancer: is it ready for primetime? JOP 2013;14:309-311.

11. Spratlin J, Sangha R, Glubrecht D, et al. The absence of human equilibrative nucleoside transporter 1 is associated with reduced survival in patients with gemcitabine-treated pancreas adenocarcinoma. Clin Cancer Res 2004;10:6956-6961.

12. Nordh S, Ansari D, Andersson R. hENT1 expression is predictive of gemcitabine outcome in pancreatic cancer: a systematic review. World J Gastroenterol 2014;20:8482-8490.

13. Farrell JJ, Elsaleh H, Garcia M, et al. Human equilibrative nucleoside transporter 1 levels predict response to gemcitabine in patients with pancreatic cancer. Gastroenterology 2009;136:187-195.

14. Greenhalf W, Ghaneh P, Neoptolemos JP, et al. Pancreatic cancer hENT1 expression and survival from gemcitabine in patients from the ESPAC-3 trial. J Natl Cancer Inst 2014;106:djt347.

15. Neoptolemos JP, Stocken DD, Bassi C, et al. Adjuvant chemotherapy with fluorouracil plus folinic acid vs gemcitabine following pancreatic cancer resection: a randomized controlled trial. JAMA 2010;304:1073-1081.

16. Poplin E, Wasan H, Rolfe L, et al. Randomized, multicenter, phase II study of CO-101 versus gemcitabine in patients with metastatic pancreatic ductal adenocarcinoma: including a prospective evaluation of the role of hENT1 in gemcitabine or CO101 sensitivity. J Clin Oncol 2013;31:4453-4461.

17. Ormanns S, Heinemann V, Raponi M, et al. Human equilibrative nucleoside transporter 1 is not predictive for gemcitabine efficacy in advanced pancreatic cancer: translational results from the AIOPK0104 phase III study with the clone SP120 rabbit antibody. Eur J Cancer 2014;50:1891-1899.

18. Heinemann V, Vehling-Kaiser U, Waldschmidt $D$, et al. Gemcitabine plus erlotinib followed by capecitabine versus capecitabine plus erlotinib followed by gemcitabine in advanced pancreatic cancer: final results of a randomised phase 3 trial of the 'Arbeitsgemeinschaft Internistische Onkologie' (AIO-PK0104). Gut 2013;62:751-759.

19. Sebastiani V, Ricci F, Rubio-Viqueira B, et al. Immunohistochemical and genetic evaluation of deoxycytidine kinase in pancreatic cancer: relationship to molecular mechanisms of gemcitabine resistance and survival. Clin Cancer Res 2006;12:2492-2497.

20. Maréchal R, Mackey JR, Lai R, et al. Deoxycitidine kinase is associated with prolonged survival after adjuvant gemcitabine for resected pancreatic adenocarcinoma. Cancer 2010;116:5200-5206.

21. Maréchal R, Bachet JB, Mackey JR, et al. Levels of gemcitabine transport and metabolism proteins predict survival times of patients treated with gemcitabine for pancreatic adenocarcinoma. Gastroenterology 2012;143:664-674.e1-e6.

22. Akita H, Zheng Z, Takeda Y, et al. Significance of RRM1 and ERCC1 expression in resectable pancreatic adenocarcinoma. Oncogene 2009;28:2903-2909.

23. Valsecchi ME, Holdbrook T, Leiby BE, et al. Is there a role for the quantification of RRM1 and ERCC1 expression in pancreatic ductal adenocarcinoma? BMC Cancer 2012;12:104.

24. Maithel SK, Coban I, Kneuertz PJ, et al. Differential expression of ERCC1 in pancreas adenocarcinoma: high tumor expression is associated with earlier recurrence and shortened survival after resection. Ann Surg Oncol 2011;18:2699-2705.

25. Mancuso A, Sacchetta S, Saletti PC, et al. Clinical and molecular determinants of survival in pancreatic cancer patients treated with second-line chemotherapy: results of an Italian/Swiss multicenter survey. Anticancer Res 2010;30:4289-4295.

26. Fuereder T, Stift J, Kuehrer I, et al. Response to GEMOX plus erlotinib in pancreatic cancer is associated with ERCC1 overexpression. Eur J Clin Invest 2014;44:958-964.

27. Takamura M, Nio Y, Yamasawa K, Dong M, Yamaguchi K, Itakura M. Implication of thymidylate synthase in the outcome of patients with invasive ductal carcinoma of the pancreas and efficacy of adjuvant chemotherapy using 5 -fluorouracil or its derivatives. Anticancer Drugs 2002;13:75-85.

28. Hu YC, Komorowski RA, Graewin S, et al. Thymidylate synthase expression predicts the response to 5-fluorouracil-based adjuvant therapy in pancreatic cancer. Clin Cancer Res 2003;9:4165-4171.

29. Formentini A, Sander S, Denzer S, Straeter J, Henne-Bruns D, Kornmann M. Thymidylate synthase expression in resectable and unresectable pancreatic cancer: role as predictive or prognostic marker? Int J Colorectal Dis 2007;22:49-55.

30. van der Zee JA, van Eijck CH, Hop WC, et al. Expression and prognostic significance of thymidylate synthase (TS) in pancreatic head and periampullary cancer. Eur J Surg Oncol 2012;38:1058-1064.

31. Nakahara $\mathrm{O}$, Takamori $\mathrm{H}$, Tanaka $\mathrm{H}$, et al. Clinical significance of dihydropyrimidine dehydrogenase and thymidylate synthase expression in patients with pancreatic cancer. Int J Clin Oncol 2010;15:39-45.

32. Kuramochi $\mathrm{H}$, Hayashi $\mathrm{K}$, Uchida $\mathrm{K}$, et al. High intratumoral dihydropyrimidine dehydrogenase mRNA levels in pancreatic cancer associated with a high rate of response to S-1. Cancer Chemother Pharmacol 2008;63:85-89.

33. Kondo N, Murakami Y, Uemura K, et al. Prognostic impact of dihydropyrimidine dehydrogenase expression on pancreatic adenocarcinoma patients treated with S-1-based adjuvant chemotherapy after surgical resection. J Surg Oncol 2011;104:146-154.

34. Kondo N, Murakami Y, Uemura K, et al. Combined analysis of dihydropyrimidine dehydrogenase and human equilibrative nucleoside transporter 1 expression predicts survival of pancreatic carcinoma patients treated with adjuvant gemcitabine plus S-1 chemotherapy after surgical resection. Ann Surg Oncol 2012;19(Suppl 3):S646-S655.

35. Nakata B, Wang YQ, Yashiro M, et al. Prognostic value of microsatellite instability in resectable pancreatic cancer. Clin Cancer Res 2002;8:2536-2540.

36. Wei Y, Xia W, Zhang Z, et al. Loss of trimethylation at lysine 27 of histone $\mathrm{H} 3$ is a predictor of poor outcome in breast, ovarian, and pancreatic cancers. Mol Carcinog 2008;47:701-706.

37. Manuyakorn A, Paulus R, Farrell J, et al. Cellular histone modification patterns predict prognosis and treatment response in resectable pancreatic adenocarcinoma: results from RTOG 9704. J Clin Oncol 2010;28:1358-1365.

38. Watanabe T, Morinaga S, Akaike M, et al. The cellular level of histone $\mathrm{H} 3$ lysine 4 dimethylation correlates with response to adjuvant gemcitabine in Japanese pancreatic cancer patients treated with surgery. Eur J Surg Oncol 2012;38:1051-1057.

39. Vaz J, Ansari D, Sasor A, Andersson R. SPARC: A Potential Prognostic and Therapeutic Target in Pancreatic Cancer. Pancreas 2015;44:1024-1035.

40. Kupprion C, Motamed K, Sage EH. SPARC (BM-40, osteonectin) inhibits the mitogenic effect of vascular endothelial growth factor 
on microvascular endothelial cells. J Biol Chem 1998;273:2963529640.

41. Gao J, Song J, Huang H, et al. Methylation of the SPARC gene promoter and its clinical implication in pancreatic cancer. J Exp Clin Cancer Res 2010;29:28.

42. Hidalgo M, Plaza C, Musteanu M, et al. SPARC expression did not predict efficacy of nab-paclitaxel plus gemcitabine or gemcitabine alone for metastatic pancreatic cancer in an exploratory analysis of the phase III MPACT trial. Clin Cancer Res 2015;21:4811-4818.

43. Hahn SA, Greenhalf B, Ellis I, et al. BRCA2 germline mutations in familial pancreatic carcinoma. J Natl Cancer Inst 2003;95:214-221.

44. Vyas $\mathrm{O}$, Leung $\mathrm{K}$, Ledbetter $\mathrm{L}$, et al. Clinical outcomes in pancreatic adenocarcinoma associated with BRCA-2 mutation. Anticancer Drugs 2015;26:224-226.

45. Bhalla A, Saif MW. PARP-inhibitors in BRCA-associated pancreatic cancer. JOP 2014;15:340-343.

46. Passero FC Jr, Grapsa D, Syrigos KN, Saif MW. The safety and efficacy of Onivyde (irinotecan liposome injection) for the treatment of metastatic pancreatic cancer following gemcitabinebased therapy. Expert Rev Anticancer Ther 2016;16:697-703.

47. Etienne-Grimaldi MC, Boyer JC, Thomas F, et al. UGT1A1 genotype and irinotecan therapy: general review and implementation in routine practice. Fundam Clin Pharmacol 2015;29:219-237.

48. Luheshi NM, Coates-Ulrichsen J, Harper J, et al. Transformation of the tumour microenvironment by a CD40 agonist antibody correlates with improved responses to PD-L1 blockade in a mouse orthotopic pancreatic tumour model. Oncotarget 2016;14:1850818520.

48. Riva F, Dronov OI, Khomenko DI, et al. Clinical applications of circulating tumor DNA and circulating tumor cells in pancreatic cancer. Mol Oncol 2016;10:481-493. 